PROCEEDINGS OF THE

AMERICAN MATHEMATICAL SOCIETY

Volume 138, Number 4, April 2010, Pages 1161-1170

S 0002-9939(09)10066-7

Article electronically published on December 2, 2009

\title{
CHARACTERIZATIONS OF THE SOLVABLE RADICAL
}

\author{
PAUL FLAVELL, SIMON GUEST, AND ROBERT GURALNICK
}

(Communicated by Jonathan I. Hall)

\begin{abstract}
We prove that there exists a constant $k$ with the property: if $\mathcal{C}$ is a conjugacy class of a finite group $G$ such that every $k$ elements of $\mathcal{C}$ generate a solvable subgroup, then $\mathcal{C}$ generates a solvable subgroup. In particular, using the Classification of Finite Simple Groups, we show that we can take $k=4$. We also present proofs that do not use the Classification Theorem. The most direct proof gives a value of $k=10$. By lengthening one of our arguments slightly, we obtain a value of $k=7$.
\end{abstract}

\section{INTRODUCTION}

The aim of this paper is to prove the following theorem:

Theorem A. There exists a constant $k$ with the property: if $\mathcal{C}$ is a conjugacy class of the finite group $G$ such that every $k$ elements of $\mathcal{C}$ generate a solvable subgroup, then $\mathcal{C}$ generates a solvable subgroup.

The most direct proof of Theorem $\mathrm{A}$ uses a value of $k=10$. The ideas involved are similar to those used in the proofs of Hall's extended Sylow Theorems together with a little representation theory. After a preprint containing a proof of Theorem $\mathrm{A}$ was circulated, Gordeev et al. [6] used the Classification of Finite Simple Groups to prove Theorem $\mathrm{A}$ with a value of $k=8$. By lengthening one of our arguments we are able to obtain a classification free proof with a value of $k=7$. Using deeper representation theory, better results are possible; see 2] and [5. As conjectured by Gordeev et al. [6], which has since been announced by them independently (see [7. 8]) we prove that, in fact, we can take $k=4$. Our result was announced in 12. Both proofs for $k=4$ rely on the Classification Theorem. Note also that 4 is best possible (consider the conjugacy class of transpositions in $S_{n}, n>4$ ). The second author, in a forthcoming paper, has classified all conjugacy classes of involutions in almost simple groups in which three conjugates always generate a solvable subgroup.

For many conjugacy classes, it is worth noting that Theorem 1.1 below implies that it is enough to consider pairs of elements in $\mathcal{C}$.

Theorem 1.1 ([10]). Let $\mathcal{C}$ be a conjugacy class of the finite group $G$ consisting of elements of prime order $p \geq 5$. Then $\mathcal{C}$ generates a solvable subgroup if and only if every pair of elements of $\mathcal{C}$ generates a solvable subgroup.

Received by the editors August 28, 2008.

2010 Mathematics Subject Classification. Primary 20F14, 20 D10.

Key words and phrases. Solvable radical, generation by conjugates.

The second and third authors were partially supported by NSF grant DMS 0653873. 
The corresponding result for nilpotency is true without any restriction on $p$. This is the Baer-Suzuki theorem, which is well known and reasonably elementary.

These results do not hold for all groups, but the finite case does yield the following results.

Corollary 1.2. Let $k$ be a field and $G$ a subgroup of $\mathrm{GL}(n, k)$.

(1) If $g \in G$, then the normal closure of $g$ in $G$ is solvable if and only if every 4 conjugates of $g$ generate a solvable subgroup.

(2) If $k$ has characteristic 0 or $p>3$ and $g \in G$ is a unipotent element, then the normal closure of $g$ in $G$ is solvable if and only if every 2 conjugates of $g$ generate a solvable subgroup.

Finally, we remark that we prove two results of independent interest. One is Lemma 3.4, where we show that if $G$ is solvable and generated by a conjugacy class $C$ and $V$ is an irreducible $G$-module, then $\operatorname{dim} C_{V}(a) \leq(3 / 4) \operatorname{dim} V$ for $a \in C$ (and this is best possible). This gives Corollary 3.5. if $V$ is a minimal normal self-centralizing subgroup of a finite solvable group and $G / V$ is generated by 5 elements in the conjugacy class $C$, then so is $G$. We also prove Theorem 2.4, which asserts that any involution in the automorphism group of a Chevalley group in odd characteristic inverts an element of odd prime order other than a long root element.

We would like to thank the referee for a careful reading of the initial version of this paper and for helpful suggestions. These included reorganizing the proof, and in particular stating Theorem 2.4 and Corollary 3.5 as separate results.

\section{Proof of Theorem A using the Classification theorem}

We use standard terminology and notation. See 1 for the basic definitions.

Let $(\mathcal{C}, G)$ be a minimal counterexample. Then every four elements of $\mathcal{C}$ generate a solvable subgroup, yet the subgroup generated by $\mathcal{C}$ is not solvable. Since $|G|$ is minimal, it is clear that the solvable radical of $G$ is trivial. The following lemma [10, Lemma 1] will be used to show that $G$ must be almost simple.

Lemma 2.1. Suppose that $G$ is a finite group such that the Fitting subgroup $F(G)$ is trivial. Let $L$ be a component of $G$.

(a) If $x$ is an element of $G$ such that $x \notin N_{G}(L)$ and $x^{2} \notin C_{G}(L)$, then there exists an element $g$ in $G$ such that $\left\langle x, x^{g}\right\rangle$ is not solvable.

(b) If $x$ is an element of $G$ such that $x \notin N_{G}(L)$ and $x^{2} \in C_{G}(L)$, then there exist elements $g_{1}$ and $g_{2}$ in $G$ such that $\left\langle x, x^{g_{1}}, x^{g_{2}}\right\rangle$ is not solvable.

Part (a) of Lemma 2.1 relies on the so-called $\frac{3}{2}$-generation result of Guralnick and Kantor [1]. Part $(b)$ of Lemma 2.1] only relies on the fact that every finite simple group can be generated by two elements (see [3]). We can now show that $G$ is almost simple:

Lemma 2.2. $G$ is almost simple.

Proof. Let $x \in \mathcal{C}$ such that every four conjugates of $x$ generate a solvable subgroup of $G$ but that $M:=\left\langle x^{G}\right\rangle$ is not solvable.

Let $N$ be a minimal normal subgroup of $G$. Since $G$ has no solvable normal subgroups, $N=L \times \cdots \times L$ with $L$ a nonabelian simple group.

By minimality, $M N / N$ is solvable. If $[x, N]=1$, then $[M, N]=1$ and so $M$ embeds in $G / N$, whence $M$ is solvable. 
TABLE 1. List of exceptions to Theorem 2.3

\begin{tabular}{|c|c|}
\hline$G_{0}$ & $x$ \\
\hline $\operatorname{PSL}(n, 3), n>2$ & transvection \\
$\operatorname{PSp}(2 n, 3), n>1$ & transvection \\
$\operatorname{PSU}(n, 3), n>2$ & transvection \\
$\operatorname{PSU}(n, 2), n>3$ & pseudoreflection of order 3 \\
$\mathrm{P} \Omega^{\epsilon}(n, 3), n>6$ & long root element \\
$E_{l}(3), F_{4}(3),{ }^{2} E_{6}(3),{ }^{3} D_{4}(3)$ & long root element \\
$G_{2}(3)$ & long or short root element \\
$G_{2}(2)^{\prime} \cong \operatorname{PSU}(3,3)$ & transvection \\
\hline
\end{tabular}

Set $H=\langle x, N\rangle$. The normal closure of $x$ in $H$ contains $[x, N]$, which is a nontrivial normal subgroup of $N$, whence is not solvable. Thus, by minimality, $G=H$, whence $x$ acts transitively on the direct factors of $N$. By Lemma 2.1, this implies that $N=L$ is simple. Since $C_{\langle x\rangle}(N)$ is central in $G$, this is trivial, whence $N$ is the unique minimal normal subgroup of $G$. Thus, $G$ is almost simple.

Let $G_{0}$ be the socle of the almost simple group $G$. The Classification of Finite Simple Groups implies that $G_{0}$ is an alternating group, a simple group of Lie type, or a sporadic group. Since the solvable radical of $G$ is trivial and $(\mathcal{C}, G)$ is a counterexample to the theorem, every four elements of $\mathcal{C}$ generate a solvable group. Observe that it suffices to assume that the elements of $\mathcal{C}$ have prime order. Indeed, the following theorem implies that we may assume that $\mathcal{C}$ is a conjugacy class of involutions. It also precludes the vast majority of possibilities for $G_{0}$.

Theorem 2.3 ([10]). Let $G$ be a finite almost simple group with socle $G_{0}$. Suppose that $x$ is an element of odd prime order in $G$. Then one of the following holds:

(i) There exists $g \in G$ such that $\left\langle x, x^{g}\right\rangle$ is not solvable.

(ii) $x^{3}=1$ and $\left(x, G_{0}\right)$ belongs to a short list of exceptions given in Table 1. Moreover, there exist $g_{1}, g_{2} \in G$ such that $\left\langle x, x^{g_{1}}, x^{g_{2}}\right\rangle$ is not solvable, unless $G_{0} \cong \operatorname{PSU}(n, 2)$ or $\operatorname{PSp}(2 n, 3)$. In any case, there exist $g_{1}, g_{2}, g_{3} \in G$ such that $\left\langle x, x^{g_{1}}, x^{g_{2}}, x^{g_{3}}\right\rangle$ is not solvable.

Now observe that if $\left\langle x, x^{g}\right\rangle$ is a 2-group for all $g \in G$, then $\left\langle x^{G}\right\rangle$ is nilpotent by the Baer-Suzuki Theorem. So if $x$ is an involution in an almost simple group there must exist a conjugate $x^{g_{1}}$ such that $\left\langle x, x^{g_{1}}\right\rangle$ is not a 2-group. Thus, $x$ inverts an element $y$ of odd prime order. So $(\mathcal{C}, G)$ cannot be a minimal counterexample unless:

(1) $G_{0}$ is one of the groups in Table 1;

(2) $\mathcal{C}$ is a conjugacy class of involutions; and

(3) if $x \in \mathcal{C}$, then $x$ inverts no elements of odd prime order other than those in the listed conjugacy classes of Table 1

Thus, the result will follow from the next two results.

We first rule out the case that $G_{0}$ is a Chevalley group over the field of 3 elements by proving the following result.

Theorem 2.4. Let $G$ be a simple group of Lie type of rank at least 2 defined over a field of odd characteristic $p$. Let $\mathcal{R}$ be the conjugacy class of long root elements 
in $G$. If $G=G_{2}\left(3^{k}\right)$, let $\mathcal{R}^{\prime}$ denote the conjugacy of short root elements of $G$. If $x \in \operatorname{Aut}(G)$ is an involution, then $x$ inverts some element $h$ of odd prime order not in $\mathcal{R}$ (or $\mathcal{R} \cup \mathcal{R}^{\prime}$ if $G=G_{2}\left(3^{k}\right)$ ).

Proof. Suppose the result is false and that $G$ is a minimal counterexample. Let $A=\langle x, G\rangle$.

By the Baer-Suzuki theorem, $x$ inverts some $z \in \mathcal{R}$. Let $P$ be the parabolic subgroup that normalizes $C_{G}(z)$. Let $L$ be a Levi subgroup of $P$ and $U$ the unipotent radical of $P$. Now observe that $N_{A}(L)$ has odd index in $N_{A}(U)$, and so a Sylow 2-subgroup of $N_{A}(U)$ is contained in $N_{A}(L)$. Thus we may assume that $x$ normalizes $L$. If $x$ centralizes $L$, then $x$ is in the central torus of $L$ (in particular, it is in a split torus). By conjugating by an element of the Weyl group, we may assume that $x$ does not centralize $[L, L]$. By minimality, it follows that either $[L, L]$ has rank 1 or $x$ inverts some $p$-element outside $\mathcal{R}$. If $[L, L]$ has rank 1 , then either $G$ has rank 2 or $G=\mathrm{SL}(4, q)$.

If $G=\mathrm{SL}(4, q)$ and $x$ induces a graph automorphism, field or field-graph automorphism, then $x$ normalizes but does not centralize an $\mathrm{SL}(3, q)$. If $x$ is an inner diagonal automorphism (of order 2), then either $x \in \operatorname{Sp}(4, q)$ or $x$ normalizes but does not centralize an $\mathrm{SL}(3, q)$. Thus, this case is eliminated by minimality.

If $G=\mathrm{Sp}(4, q), \mathrm{SU}(4, q)$ or $\mathrm{SU}(5, q)$, then $x$ acts on $Q:=\mathcal{O}_{p}\left(C_{G}(z)\right)$, the largest normal $p$-subgroup of $C_{G}(z)$. This is a special group with commutator and center both equal to the root subgroup containing $z$. Moreover, all root elements in $Q$ are contained in $Z(Q)$. Since $x$ does not centralize $Z(Q)$, it cannot centralize $Q / Z(Q)$ and so it inverts some element of $Q \backslash Z(Q)$, and this contradicts the choice of $x$.

Suppose that $G=\mathrm{SL}(3, q)$. If $x$ is a field, graph or field-graph automorphism, then we can choose $x$ to normalize a maximal torus of order $\left(q^{3}-1\right) /(q-1)$ and so it will invert some semisimple element of odd order. The one remaining conjugacy class of involutions is the unique conjugacy class of inner-diagonal involutory automorphisms. It is straightforward to see that $x$ inverts a regular unipotent element of order $p$.

Suppose that $G=G_{2}(q)$. Note that if $3 \mid q$, then $x$ cannot involve a graph automorphism (for then $x$ interchanges the conjugacy classes of long and short roots). So in all cases, we may assume that $x$ is in the group generated by inner and field automorphisms. Now $G$ contains subgroups $H$ of the form $\operatorname{SU}(3, q)$ and $\mathrm{SL}(3, q)$. Moreover, the normalizer of one of these will contain a Sylow 2-subgroup of $A$. So we may assume that our involution normalizes $H$ (and does not centralize $H$ since the centralizer of $H$ has odd order). By minimality, $x$ inverts some element of odd prime order in $H$ (which is not a long root element in $H$ and so not a root element in $G_{2}$ ).

The only remaining case to deal with is $G={ }^{3} D_{4}(q)$. We can reduce to one of $\operatorname{PGL}(3, q), \operatorname{PGU}(3, q)$ whose normalizer contains a Sylow 2-subgroup of $\operatorname{Aut}(G)$.

Next we rule out the case that $G=\operatorname{PSU}(n, q)$ with $n>3$ and $q=2$.

Lemma 2.5. Let $G=\operatorname{PSU}(n, 2), n>3$. If $x \in \operatorname{Aut}(G)$ is an involution, then $x$ inverts an element of odd order other than a pseudoreflection.

Proof. We can view any involution as acting semilinearly on the natural module for $\mathrm{GU}(n, q)$. If the result fails, then by Baer-Suzuki, $x$ inverts some pseudoreflection and therefore normalizes $H:=\mathrm{SU}(n-1, q)$. Since the centralizer of $H$ has order dividing 3 , we may assume that $x$ normalizes but does not centralize $H$. 
So the result follows by induction once we have handled the case $G=\mathrm{SU}(4,2)$. Since $\operatorname{PSU}(4,2) \cong \operatorname{PSp}(4,3)$ and pseudoreflections correspond to root elements, the previous result applies.

\section{A ClASSIFICATION FREE APPROACH}

The purpose of this section is to explore what can be proved using only elementary means.

For $G$ a nontrivial solvable group, we let $f(G)$ denote the Fitting height of $G$. This is the smallest integer $n$ such that $G$ possesses a series

$$
1=F_{0} \unlhd F_{1} \unlhd \cdots \unlhd F_{n}=G
$$

with $F_{i+1} / F_{i}$ nilpotent for all $i$. The trivial group has Fitting height 0 ; a nontrivial nilpotent group has Fitting height 1 ; and if $G \neq 1$, then $f(G / F(G))=f(G)-1$.

If $G \neq 1$ is solvable we define

$$
\psi(G)=\bigcap\{K \unlhd G \mid f(G / K)<f(G)\} .
$$

Now $G / \psi(G)$ is isomorphic to a subgroup of a direct product of groups each with Fitting height less than $f(G)$. Thus $f(G / \psi(G))<f(G)$. It follows that

$$
1 \neq \psi(G) \leq F(G)
$$

and that $\psi(G)$ is the unique smallest normal subgroup of $G$ such that the corresponding quotient group has Fitting height less than the Fitting height of $G$.

Lemma 3.1. Let $H$ be a subgroup of the solvable group $G \neq 1$. If $f(H)=f(G)$, then

$$
\psi(H) \leq \psi(G) \leq F(G) .
$$

Proof. Set $\bar{G}=G / \psi(G)$, so that $f(\bar{G})<f(G)$. Then $f(\bar{H}) \leq f(\bar{G})<f(G)=$ $f(H)$, so the definition of $\psi(H)$ implies that $\psi(H) \leq \psi(G)$. We have already seen that $\psi(G) \leq F(G)$.

Lemma 3.2. Let $G$ be a solvable group, let $N \unlhd G$, set $\bar{G}=G / N$ and suppose that $\bar{G} \neq 1$. Then the following are equivalent:

(i) $\overline{\psi(G)} \neq 1$.

(ii) $f(\bar{G})=f(G)$.

(iii) $\psi(\bar{G})=\overline{\psi(G)}$.

Proof. Suppose that $\overline{\psi(G)} \neq 1$. Then $\psi(G) \not \leq N$, so the definition of $\psi(G)$ implies that $f(\bar{G})=f(G)$. Thus (i) implies (ii).

Suppose that $f(\bar{G})=f(G)$. Now $\bar{G} / \overline{\psi(G)}$ is a homomorphic image of $G / \psi(G)$, so $f(\bar{G} / \overline{\psi(G)}) \leq f(G / \psi(G))<f(G)=f(\bar{G})$, whence $\psi(\bar{G}) \leq \overline{\psi(G)}$. Let $K$ be the full inverse image of $\psi(\bar{G})$ in $G$. Then $G / K \cong \bar{G} / \psi(\bar{G})$ so $f(G / K)<f(\bar{G})=f(G)$, whence $\psi(G) \leq K$ and then $\overline{\psi(G)} \leq \bar{K}=\psi(\bar{G})$. We deduce that $\psi(\bar{G})=\overline{\psi(G)}$. Thus (ii) implies (iii).

Since $\bar{G} \neq 1$ we have $\psi(\bar{G}) \neq 1$, so (iii) implies (i).

The next lemma is well known.

Lemma 3.3. Suppose that the solvable group $G$ possesses a unique minimal normal subgroup $V$. If $V$ has a complement in $G$, then $V$ acts transitively by conjugation on the set of complements to $V$ in $G$. 
Proof. Suppose that $A$ is a complement to $V$ and let $Q$ be a minimal normal subgroup of $A$, so that $Q$ is a $q$-group for some prime $q$. Set $K=Q V$. Now $C_{V}(Q)=1$ since otherwise $Q$ would be another minimal normal subgroup of $G$. It follows that $V$ is an $r$-group for some prime $r \neq q$. Then $Q$ is a Sylow $q$-subgroup of $K$, and any complement to $V$ in $G$ is the normalizer of a Sylow $q$-subgroup of $K$. The result now follows from Sylow's Theorem.

The following extends a result that appears in [17, page 82]. One can construct examples to see that the result is best possible (take $G$ to be a subgroup of the monomial subgroup of GL $(4, k)$ with $a$ a transposition in $\left.S_{4} \leq G\right)$.

Lemma 3.4. Let $G$ be a solvable group that possesses an element a such that $G=\left\langle a^{G}\right\rangle$. Let $k$ be a field. Let $V$ be a nontrivial irreducible $k G$-module. Then

$$
\operatorname{dim} C_{V}(a) \leq \frac{3}{4} \operatorname{dim} V .
$$

Proof. First note that by replacing $k$ by $\operatorname{End}_{k G}(V)$, we may assume that $V$ is absolutely irreducible. Then we can extend scalars and assume that $k$ is algebraically closed. Clearly, we may assume that $G$ acts faithfully on $V$.

Assume false, so that $\operatorname{dim} C_{V}(a)>\frac{3}{4} \operatorname{dim} V$. We will construct a normal subgroup of $G$ that has more than one homogeneous component on $V$. The proof then proceeds by analyzing the permutation action of $G$ on those components.

Let $g, h \in G$. The subspaces $C_{V}(a)$ and $C_{V}\left(a^{g}\right)$ both have dimension greater than $\frac{3}{4} \operatorname{dim} V$, so their intersection has dimension greater than $\frac{1}{2} \operatorname{dim} V$. Since $[g, a]$ acts trivially on this intersection it follows that

$$
\operatorname{dim} C_{V}([g, a])>\frac{1}{2} \operatorname{dim} V .
$$

Repeating this argument, we deduce that

$$
C_{V}([g, a]) \neq 0 \text { and } C_{V}([g, a, h]) \neq 0
$$

for all $g, h \in G$.

Since $G$ acts irreducibly and faithfully on $V$ we have

$$
C_{V}(z)=0
$$

for all $z \in Z(G)^{\#}$. In particular, $a \notin Z(G)$. Let $N$ be a normal subgroup of $G$ chosen minimal subject to the fact that $N$ is not central in $G$. Now $N$ is solvable, so $N^{\prime}<N$, whence $N^{\prime} \leq Z(G)$. We claim that $N$ is abelian. If not, then $Z(N)<N$, whence $Z(N) \leq Z(G)$.

Since $G=\left\langle a^{G}\right\rangle$ and $N$ is not central, we see that $[a, N] \neq 1$. Choose $g \in N$ such that $[g, a] \neq 1$. Since $[g, a]$ fixes a nonzero vector in $V,[g, a]$ is a noncentral element of $N$. Now choose $h \in N$ with $1 \neq[g, a, h] \in N^{\prime} \leq Z(G)$. Thus, $[g, a, h]$ is a nontrivial scalar on $V$, but by Equations 1 and 2 , this is not the case. Thus, $N$ is abelian.

Since $N$ is abelian and not central in $G, V=V_{1} \oplus \ldots \oplus V_{r}$ is a direct sum of the $N$ eigenspaces $V_{i}$ with $r>1$. Set $\Omega=\left\{V_{1}, \ldots, V_{r}\right\}$. Since $V$ is irreducible, $G$ acts transitively on $\Omega$. Since $G$ is generated by the conjugates of $a, a$ acts nontrivially on $\Omega$. Set $e=\operatorname{dim} V_{i}$.

We claim that $a$ fixes no more than $d / 2$ points in any transitive permutation action of $G$ of degree $d>1$. It suffices to prove this for a primitive action (if $a$ fixes no more than $1 / 2$ the blocks, it fixes no more than $1 / 2$ the points). In any 
primitive action of $G, a$ acts nontrivially. Since a primitive permutation action of a finite solvable group consists of affine transformations of a vector space over a prime field, the claim follows.

If $\Delta$ is an $a$-orbit on $\Omega$ and $V_{\Delta}=\sum_{i \in \Delta} V_{i}$, then $\operatorname{dim} C_{V_{\Delta}}(a) \leq e$. Thus, $\operatorname{dim} C_{V}(a) \leq e f$, where $f$ is the number of orbits of $a$ on $\Omega$. Since $a$ fixes at most $r / 2$ points, it has at most $3 r / 4$ orbits on $\Omega$, whence $\operatorname{dim} C_{V}(a) \leq(3 / 4) \operatorname{dim} V$, a contradiction.

We point out the following corollary.

Corollary 3.5. Let $G$ be a finite solvable group with $V$ a minimal normal selfcentralizing subgroup of $G$. If $G=\left\langle a_{1}, \ldots, a_{5}, V\right\rangle$ with the $a_{i}$ all conjugate to $a$, then $G=\left\langle b_{1}, \ldots, b_{5}\right\rangle$, where each $b_{j}$ is conjugate to a.

Proof. Clearly, $G=\left\langle a^{G}\right\rangle$ (since $\left\langle a^{G}\right\rangle$ is a normal subgroup and acts irreducibly and nontrivially on $V$, whence it contains $V$ ). Now apply Lemma 3.4 to conclude that $\operatorname{dim} C_{V}(a) \leq(3 / 4) \operatorname{dim} V$. Choose $v_{i} \in\left[a_{i}, V\right]$. Note that $a_{i} v_{i}$ is conjugate to $a_{i}$ and so to $a$. Let $H\left(v_{1}, \ldots, v_{5}\right)=\left\langle a_{1} v_{1}, \ldots, a_{5} v_{5}\right\rangle$. Note that any such subgroup covers $G / V$, and so either the result follows or each $H\left(v_{1}, \ldots, v_{5}\right)$ is a complement to $V$ in $G$.

Assume that this is the case. Note that the map $\left(v_{1}, \ldots, v_{5}\right) \rightarrow H\left(v_{1}, \ldots, v_{5}\right)$ is an injection (for if $a_{i} v_{i}$ and $a_{i} v_{i}^{\prime}$ are both in $H\left(v_{1}, \ldots, v_{5}\right)$, then $V \cap H\left(v_{1}, \ldots, v_{5}\right) \neq 1$ and so $V \leq H\left(v_{1}, \ldots, v_{5}\right)$, whence $\left.H\left(v_{1}, \ldots, v_{5}\right)=G\right)$.

Thus, the number of complements to $V$ is at least $|[a, V]|^{5} \geq|V|^{5 / 4}$. On the other hand, the number of complements to $V$ is at most $|V|$ by Lemma 3.3. This contradiction completes the proof.

Lemma 3.6. Let $G$ be a solvable group and let a be an element of $G$. Suppose that $A$ is a subgroup of $G$ with the following properties:

(i) $A=\left\langle a_{1}, \ldots, a_{5}\right\rangle$, where $a_{1}, \ldots, a_{5}$ are conjugate to a in $G$ and conjugate to one another in $A$.

(ii) A has maximal Fitting height subject to (i).

Then

$$
\psi(A) \leq F(G)
$$

Proof. Assume false and let $G$ be a minimal counterexample, so that $\psi(A) \not \leq F(G)$. Let $V$ be a minimal normal subgroup of $G$ and set

$$
\bar{G}=G / V
$$

Now $V$ is abelian, so $V \leq F(G)$. In particular, $\psi(A) \not \leq V$, and then the definition of $\psi(A)$ implies that

$$
f(\bar{A})=f(A) .
$$

We claim that $\bar{A}$ satisfies (i) and (ii) when $G$ is replaced by $\bar{G}$ and $a$ by $\bar{a}$. Certainly (i) is satisfied. As for (ii), let $\bar{B}$ be a subgroup of $\bar{G}$ such that $\bar{B}=$ $\left\langle\bar{b}_{1}, \ldots, \bar{b}_{5}\right\rangle$ with $\bar{b}_{1}, \ldots, \bar{b}_{5}$ conjugate to $\bar{a}$ in $\bar{G}$ and conjugate to one another in $\bar{B}$. Let $b_{1}$ be a conjugate of $a$ that maps onto $\bar{b}_{1}$ and let $B$ be an inverse image of $\bar{B}$ that is minimal subject to $b_{1} \in B$. Choose $g_{2}, \ldots, g_{5} \in B$ such that $\bar{b}_{1}^{\bar{g}_{i}}=\bar{b}_{i}$. Then $\left\langle b_{1}, b_{1}^{g_{2}}, \ldots, b_{1}^{g_{5}}\right\rangle$ is a subgroup of $B$ that maps onto $\bar{B}$. The minimality of $B$ forces 
$B=\left\langle b_{1}, b_{1}^{g_{2}}, \ldots, b_{1}^{g_{5}}\right\rangle$ and as $g_{2}, \ldots, g_{5} \in B$ we see that $B$ is a subgroup of $G$ that satisfies (i). Consequently

$$
f(A) \geq f(B) .
$$

Now $\bar{B}$ is a homomorphic image of $B$, so $f(B) \geq f(\bar{B})$, and then using (3) we have

$$
f(\bar{A}) \geq f(\bar{B}) .
$$

This proves the claim.

The minimality of $G$ and the previous paragraph imply that $\psi(\bar{A}) \leq F(\bar{G})$. Lemma 3.2 and (3) imply that $\psi(\bar{A})=\overline{\psi(A)}$, so we deduce that

$$
\overline{\psi(A)} \leq F(\bar{G})
$$

It follows readily that $V$ is the unique minimal normal subgroup of $G$. Indeed, if $U$ were another such subgroup, then $\left\langle\psi(A)^{G}\right\rangle$ would embed into the nilpotent group $F(G / U) \times F(G / V)$, contrary to the fact that $\psi(A) \not \leq F(G)$.

Since $\psi(A)$ and $F(G)$ are nilpotent and since $\psi(A) \not \leq F(G)$, there exists a prime $q$ such that $\mathcal{O}_{q}(\psi(A)) \not \leq \mathcal{O}_{q}(G)$. Set $Q=\mathcal{O}_{q}(\psi(A))$. By (4) we have $\bar{Q} \leq \mathcal{O}_{q}(\bar{G})$. Let $K$ be the full inverse image of $\mathcal{O}_{q}(\bar{G})$ in $G$, so that $Q \leq K \unlhd G$ and $K / V$ is a $q$-group. Now $G$ is solvable, so $V$ is an elementary abelian $r$-group for some prime $r$. Moreover, $Q \not \leq \mathcal{O}_{q}(G)$, so $K$ is not a $q$-group and hence $r \neq q$. Since $V$ is the unique minimal normal subgroup of $G$, we deduce that $\mathcal{O}_{q}(G)=1$.

We claim that

$$
C_{K}(V)=V .
$$

Indeed, choose $S \in \operatorname{Syl}_{q}(K)$. Since $K / V$ is a $q$-group we have $K=S V$, whence $C_{K}(V)=C_{S}(V) \times V$. Then $C_{S}(V)=\mathcal{O}_{q}\left(C_{K}(V)\right) \leq \mathcal{O}_{q}(K) \leq \mathcal{O}_{q}(G)=1$, proving the claim.

Suppose that $A V \neq G$. Then the minimality of $G$ implies that $Q \leq \mathcal{O}_{q}(A V)$, so as $V \leq \mathcal{O}_{r}(A V)$ we see that $[Q, V]=1$, contrary to (5). We deduce that

$$
G=A V \text {. }
$$

If $f(A)=f(G)$, then Lemma 3.1 implies that $Q \leq \mathcal{O}_{q}(G)$, contrary to the choice of $q$. Thus $f(A)<f(G)$, and then the definition of $A$ implies that $G$ cannot be generated by 5 conjugates of $a$. Moreover, we have $A \neq G$, so using (6) and the fact that $V$ is a minimal normal subgroup of $G$ we deduce that $A \cap V=1$, so $A$ is a complement to $V$ in $G$.

Since $A=\left\langle a_{1}, \ldots, a_{5}\right\rangle$, Corollary 3.5 implies that $G$ can be generated by 5 conjugates of $a$, a contradiction.

The following lemma proves Theorem A.

Lemma 3.7. Let $\mathcal{C}$ be a conjugacy class of the group $G$. If every 10 members of $\mathcal{C}$ generate a solvable subgroup, then $\mathcal{C}$ generates a solvable subgroup.

Proof. Assume false and let $G$ be a minimal counterexample. Then $G$ possesses no nontrivial normal solvable subgroups, and we may suppose that the elements of $\mathcal{C}$ have prime order. Let $a \in \mathcal{C}$ and let $A$ be a subgroup of $G$ that satisfies

(1) $A=\left\langle a_{1}, \ldots, a_{5}\right\rangle$, where $a_{1}, \ldots, a_{5}$ are conjugate to $a$ in $G$ and conjugate to one another in $A$, and

(2) $A$ has maximal Fitting height subject to (1). 
Replacing $A$ by a suitable conjugate, we may suppose that $a_{1}=a$. Let $Q=\psi(A)$, so that $Q \neq 1$. Let $g \in G$ and set $H=\left\langle A, A^{g}\right\rangle$. By hypothesis, $H$ is solvable, so Lemma 3.6 with $H$ in place of $G$ yields $Q \leq F(H)$. Similarly, $Q^{g} \leq F(H)$. We deduce that $\left\langle Q, Q^{g}\right\rangle$ is nilpotent for all $g \in G$. The Baer-Suzuki Theorem implies that

$$
Q \leq F(G)
$$

This contradicts the fact that $G$ has no nontrivial normal solvable subgroups and completes the proof.

Using a slightly longer argument we are able to replace 10 by 7 . First we need:

Lemma 3.8. Suppose $A \neq 1$ is solvable and that $\psi(A) \leq Z(A)$. Then $A$ is abelian.

Proof. We have

$$
f(A / \psi(A))<f(A) .
$$

On the other hand, for any $n \geq 1$, the class of solvable groups of Fitting height $n$ is closed under central extensions. This forces $f(A / \psi(A))=0$, whence $A=\psi(A)$. As $\psi(A) \leq Z(A)$, the conclusion follows.

Theorem 3.9. Let $\mathcal{C}$ be a conjugacy class of the group $G$. If every 7 members of $\mathcal{C}$ generate a solvable subgroup, then $\mathcal{C}$ generates a solvable subgroup.

Proof. Proceed as in the proof of the previous lemma and construct the subgroup $A$.

We claim there is a prime $p$, a conjugate $b$ of $a$ and a $p$-subgroup $P$ with $1 \neq$ $P \leq \psi(A) \cap\langle a, b\rangle$. If $[\psi(A), a] \neq 1$ there exists a prime $p$ and $x \in \mathcal{O}_{p}(\psi(A))$ with $[a, x] \neq 1$. Put $b=a^{x}$ and $P=\langle[a, x]\rangle$. Suppose that $[\psi(A), a]=1$. As $A=\left\langle a^{A}\right\rangle$ it follows that $\psi(A) \leq Z(A)$. The previous lemma implies that $A$ is abelian. Then $A=\langle a\rangle$. Put $b=a$ and $P=A$.

Let $g \in G$ and set $H=\left\langle A, a^{g}, b^{g}\right\rangle$. By hypothesis, $H$ is solvable. Lemma 3.6 implies $P \leq \mathcal{O}_{p}(H)$. As $P^{g} \leq\langle a, b\rangle^{g} \leq H$ it follows that $\left\langle P, P^{g}\right\rangle$ is a $p$-group. A contradiction follows from the Baer-Suzuki Theorem.

\section{Proof of the corollary}

The proof of Corollary 1.2 is standard. We first prove (1). We first note the wellknown fact that if $H$ is a solvable subgroup of $\operatorname{GL}(n, k)$, then the derived length of $H$ is bounded by a function $f=f(n)$.

So suppose that the normal closure $N$ of $g$ in $H$ is not solvable. Then there is some nontrivial element $x$ in the $f$ th term in the derived series of $N$. We may pass to a subgroup of $G$ and assume that $G$ is finitely generated, and so $G \leq \mathrm{GL}(n, R)$, where $R$ is a finitely generated ring over the prime field of $k$. We can choose a maximal ideal $M$ of $R$ such that $x$ is not in the congruence kernel of the map $\phi: \operatorname{GL}(n, R) \rightarrow \mathrm{GL}(n, R / M)$. Thus, $\phi(N)$ is not solvable and $\phi(G)$ is finite, whence some four conjugates of $\phi(g)$ generate a nonsolvable subgroup. Thus, the same is true for $G$.

The proof of (2) is essentially the same. First, as above, reduce to the case that $G$ is finitely generated and contained in $\operatorname{GL}(n, R)$, where $R$ is a finitely generated ring over $\mathbb{Z}$. Now argue exactly as above (except that if the characteristic is 0 , take $M$ to be a maximal ideal containing some prime $p>3$ ), and so our unipotent element in the image has order divisible by the characteristic, a prime at least 5 . 


\section{REFERENCES}

[1] M. Aschbacher, Finite group theory, second edition. Cambridge Studies in Advanced Mathematics, 10, Cambridge University Press, Cambridge, 2000. MR.1777008 (2001c:20001)

[2] A. Al-Roqi and P. Flavell, On the Fitting height of a solvable group that is generated by a conjugacy class of 3-elements. Bull. Lond. Math. Soc. 39 (2007), part 6, 973-981. MR 2392820 (2008m:20030)

[3] M. Aschbacher and R. Guralnick, Some applications of the first cohomology group, J. Algebra 90 (1984), no. 2, 446-460. MR760022 (86m:20060)

[4] J. H. Conway, R. T. Curtis, S. P. Norton, R. A. Parker, and R. A. Wilson, Atlas of finite groups, in Maximal subgroups and ordinary characters for simple groups, With computational assistance from J. G. Thackray, Oxford University Press, Eynsham, 1985. MR827219 (88g:20025)

[5] P. Flavell, On the Fitting height of a solvable group that is generated by a conjugacy class. J. London Math. Soc. (2) 66 (2002), 101-113. MR.1911223 (2003f:20024)

[6] N. Gordeev, F. Grunewald, B. Kunyavskii, and E. Plotkin, On the number of conjugates defining the solvable radical of a finite group, C. R. Acad. Sci. Paris, Ser. I 343 (2006), 387-392. MR2259878 (2007f:20032)

[7] N. Gordeev, F. Grunewald, B. Kunyavskii, and E. Plotkin, A description of Baer-Suzuki type of the solvable radical of a finite group, J. Pure and Applied Algebra 213 (2009), 250-258. MR2467402 (2009i:20045)

[8] N. Gordeev, F. Grunewald, B. Kunyavskii, and E. Plotkin, Baer-Suzuki Theorem for the solvable radical of a finite group, preprint.

[9] Daniel Gorenstein, Richard Lyons, and Ronald Solomon, The classification of the finite simple groups. Number 3, Mathematical Surveys and Monographs, vol. 40, American Mathematical Society, Providence, RI, 1998. MR1490581 (98j:20011)

[10] Simon Guest, A solvable version of the Baer-Suzuki theorem, Trans. Amer. Math. Soc., to appear.

[11] Robert M. Guralnick and William M. Kantor, Probabilistic generation of finite simple groups, J. Algebra 234 (2000), no. 2, 743-792, Special issue in honor of Helmut Wielandt. MR 1800754 (2002f:20038)

[12] R. Guralnick, E. Plotkin and A, Shalev, Burnside-type problems related to solvability, Internat. J. Algebra Comput. 17 (2007), 1033-1048. MR2355682

[13] Robert M. Guralnick and Jan Saxl, Generation of finite almost simple groups by conjugates, J. Algebra 268 (2003), no. 2, 519-571. MR2009321 (2005f:20057)

[14] Martin W. Liebeck, The classification of finite simple Moufang loops, Math. Proc. Cambridge Philos. Soc. 102 (1987), no. 1, 33-47. MR886433 (88g:20146)

[15] Martin W. Liebeck and Jan Saxl, Minimal degrees of primitive permutation groups, with an application to monodromy groups of covers of Riemann surfaces, Proc. London Math. Soc. (3) 63 (1991), no. 2, 266-314. MR.1114511 (92f:20003)

[16] G. Malle, J. Saxl, and T. Weigel, Generation of classical groups, Geom. Dedicata 49 (1993), no. 1, 85-116. MR.1261575 (95c:20068)

[17] O. Manz and T.R. Wolf, Representations of Solvable Groups. London Math. Soc. Lecture Note Series, 185, Cambridge University Press, 1993. MR.1261638 (95c:20013)

School of Mathematics, University of Birmingham, Birmingham B15 2TT, United KINGDOM

E-mail address: P.J.Flavell@bham.ac.uk

Department of Mathematics, Baylor University, One Bear Place \#97328, Waco, TEXas 76798-7328

E-mail address: Simon_Guest@baylor.edu

Department of Mathematics, University of Southern California, Los Angeles, CalIFORNIA 90089-2532

E-mail address: guralnic@usc.edu 